\title{
Neutrino analysis of the September 2010 Crab Nebula flare and time-integrated constraints on neutrino emission from the Crab using IceCube.
}

R. Abbasi ${ }^{\text {ab }}$, Y. Abdou , T. Abu-Zayyad ${ }^{\mathrm{ag}}$, J. Adams $^{\mathrm{p}}$, J. A. Aguilar ${ }^{\mathrm{ab}}$, M. Ahlers ${ }^{\text {af }}$, D. Altmann ${ }^{\mathrm{a}}$, K. Andeen ${ }^{\mathrm{ab}}$, J. Auffenberg ${ }^{\mathrm{al}}$, X. Baia ${ }^{\mathrm{ae}}$, M. Baker ${ }^{\mathrm{ab}}$, S. W. Barwick ${ }^{\mathrm{x}}$, R. Bay ${ }^{\mathrm{g}}$, J. L. Bazo Alba ${ }^{\mathrm{am}}$, K. Beattie ${ }^{\mathrm{h}}$, J. J. Beatty ${ }^{\mathrm{r}, \mathrm{s}}, \mathrm{S}$. Bechet ${ }^{\mathrm{m}}$, J. K. Becker ${ }^{\mathrm{j}}$, K.-H. Becker ${ }^{\text {al }}$,

M. L. Benabderrahmane ${ }^{\text {am }}, \mathrm{S}$. BenZvi $^{\text {ab }}$, J. Berdermann ${ }^{\text {am }}$, P. Berghaus ${ }^{\text {ae }}$, D. Berley ${ }^{\mathrm{q}}$, E. Bernardinia ${ }^{\mathrm{am}}$, D. Bertrand ${ }^{\mathrm{m}}$, D. Z. Besson ${ }^{\mathrm{z}}$, D. Bindigal, M. Bissok ${ }^{\text {a }}$, E. Blaufuss ${ }^{q}$, J. Blumenthal ${ }^{\mathrm{a}}$, D. J. Boersma ${ }^{\mathrm{a}}$, C. Bohm ${ }^{\text {ah }}$, D. Bose ${ }^{\mathrm{n}}$, S. Böser ${ }^{\mathrm{k}}$, O. Botner ${ }^{\text {ak }}$, A. M. Brown ${ }^{\mathrm{p}}$, S. Buitink ${ }^{\mathrm{n}}$,

K. S. Caballero-Mora aj, M. Carsonv ${ }^{\mathrm{v}}$, D. Chirkin ${ }^{\mathrm{ab}}$, B. Christy ${ }^{\mathrm{q}}$, J. Clem ${ }^{\mathrm{ae}}$, F. Clevermann ${ }^{\mathrm{t}}$, S. Cohen ${ }^{\mathrm{y}}$, C. Colnard ${ }^{\mathrm{w}}$, D. F. Cowen ${ }^{\text {aj,ai }}$, M. V. D'Agostino ${ }^{g}$, M. Danninger ${ }^{\text {ah }}$, J. Daughhetee ${ }^{\mathrm{e}}$, J. C. Davis ${ }^{\mathrm{r}}$, C. De Clercq ${ }^{n}$, L. Demirörs ${ }^{y}$, T. Denger ${ }^{k}$, O. Depaepe ${ }^{n}$, F. Descamps ${ }^{v}$, P. Desiati ${ }^{\mathrm{ab}}$, G. de Vries-Uiterweerd ${ }^{\mathrm{v}}$, T. DeYoung ${ }^{\mathrm{aj}}$, J. C. Díaz-Vélez ${ }^{\mathrm{ab}}$, M. Dierckxsens ${ }^{\mathrm{m}}$, J. Dreyer ${ }^{\mathrm{j}}$, J. P. Dumm ${ }^{\text {ab }}$, R. Ehrlich ${ }^{\mathrm{q}}$, J. Eisch ${ }^{\mathrm{ab}}$, R. W. Ellsworth ${ }^{\text {, }}$ O. Engdegård ${ }^{\text {ak }}$, S. Euler ${ }^{\text {a }}$, P. A. Evenson ${ }^{\text {ae }}$, O. Fadiran ${ }^{\mathrm{d}}$, A. R. Fazely ${ }^{\mathrm{f}}$, A. Fedynitch ${ }^{\mathrm{j}}$, J. Feintzeigab ${ }^{\mathrm{a}}$, T. Feusels ${ }^{\mathrm{v}}$, K. Filimonov ${ }^{\mathrm{g}}$, C. Finley ${ }^{\text {ah }}$, T. Fischer-Wasels ${ }^{\text {al }}$, M. M. Foerster ${ }^{\text {aj }}$, B. D. Fox ${ }^{\text {aj, }}$, A. Franckowiak ${ }^{k}$, R. Franke ${ }^{\text {am }}$, T. K. Gaisser ${ }^{\text {ae }}$, J. Gallagher ${ }^{\text {aa }}$, L. Gerhardt ${ }^{\mathrm{h}, \mathrm{g}}$, L. Gladstone ${ }^{\mathrm{ab}}$, T. Glüsenkamp ${ }^{\mathrm{a}}$, A. Goldschmidt ${ }^{\mathrm{h}}$, J. A. Goodman ${ }^{\mathrm{q}}$, D. Gora ${ }^{\mathrm{am}}$, D. Grant ${ }^{\mathrm{u}}$, T. Griesel ${ }^{\mathrm{ac}}$, A. Groß ${ }^{\mathrm{p}, \mathrm{w}}$, S. Grullon ${ }^{\text {ab }}$, M. Gurtner ${ }^{\text {al }}$, C. Ha ${ }^{\text {aj }}$, A. Hajismail ${ }^{v}$, A. Hallgren ${ }^{\text {ak }}$, F. Halzen ${ }^{\mathrm{ab}}$, K. Han ${ }^{\mathrm{am}}$, K. Hanson ${ }^{\mathrm{m}, \mathrm{ab}}$, D. Heinen ${ }^{\mathrm{a}}$, K. Helbing ${ }^{\mathrm{al}}$, P. Herquet ${ }^{\text {ad }}$, S. Hickford ${ }^{p}$, G. C. Hill ${ }^{\text {ab }}$, K. D. Hoffman ${ }^{\mathrm{q}}$, A. Homeierk ${ }^{\mathrm{k}}$, K. Hoshina ${ }^{\text {ab }}$, D. Hubert ${ }^{n}$, W. Huelsnitz ${ }^{\mathrm{q}}$, J.-P. Hülß ${ }^{\mathrm{a}}$, P. O. Hulth ${ }^{\text {ah }}$, K. Hultqvist ${ }^{\text {ah }}$, S. Hussain ${ }^{\text {ae }}$, A. Ishihara ${ }^{\circ}$, J. Jacobsen ${ }^{\text {ab }}$, G. S. Japaridze ${ }^{\text {d }}$, H. Johansson ${ }^{\text {ah }}$, J. M. Joseph ${ }^{\text {h }}$ K.-H. Kampert ${ }^{\text {al }}$, A. Kappes ${ }^{\text {i }}$ T. Karg ${ }^{\text {al }}$,

A. Karle ${ }^{\mathrm{ab}}$, P. Kenny ${ }^{\mathrm{z}}$, J. Kiryluk ${ }^{\text {h,g }}$, F. Kislat ${ }^{\mathrm{am}}$, S. R. Klein ${ }^{\text {h,g }}$, J.-H. Köhne ${ }^{t}$, G. Kohnen ${ }^{\text {ad }}$, H. Kolanoski ${ }^{i}$, L. Köpke ${ }^{\text {ac }}$, S. Kopper ${ }^{\text {al }}$, D. J. Koskinen ${ }^{\text {aj }}$, M. Kowalski ${ }^{\mathrm{k}}$, T. Kowarik ${ }^{\text {ac }}$, M. Krasberg ${ }^{\text {ab }}$, T. Krings ${ }^{\mathrm{a}}$,

\footnotetext{
${ }^{1}$ also Università di Bari and Sezione INFN, Dipartimento di Fisica, I-70126, Bari, Italy

${ }^{2}$ NASA Goddard Space Flight Center, Greenbelt, MD 20771, USA
} 
G. Krollac ${ }^{a c}$ N. Kurahashi ${ }^{\mathrm{ab}}$, T. Kuwabara ${ }^{\mathrm{ae}}$, M. Labare $^{\mathrm{n}}$, S. Lafebre ${ }^{\mathrm{aj}}$, K. Laihem ${ }^{\mathrm{a}}$, H. Landsman ${ }^{\mathrm{ab}}$, M. J. Larson ${ }^{\mathrm{aj}}$, R. Lauer ${ }^{\mathrm{am}}$, J. Lünemann ${ }^{\mathrm{ac}}$,

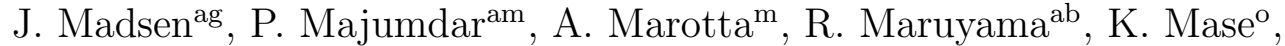
H. S. Matis ${ }^{\text {h }}$ K. Meagher ${ }^{\mathrm{q}}$, M. Merck ${ }^{\mathrm{ab}}$, P. Mészáros ${ }^{\mathrm{ai}, a j}$, T. Meures ${ }^{\mathrm{m}}$, E. Middellam ${ }^{\mathrm{am}}$ N. Milke ${ }^{\mathrm{t}}$, J. Miller ${ }^{\mathrm{ak}}$, T. Montaruli ${ }^{\mathrm{ab}, 1}$, R. Morse ${ }^{\mathrm{ab}}$, S. M. Movit ${ }^{\text {ai }}$, R. Nahnhauer ${ }^{\mathrm{am}}$, J. W. Nam ${ }^{\mathrm{x}}$, U. Naumann ${ }^{\text {al }}$, P. Nießen ${ }^{\text {ae }}$, D. R. Nygren ${ }^{\mathrm{h}}$, S. Odrowski ${ }^{\mathrm{w}}$, A. Olivas ${ }^{\mathrm{q}}$, M. Olivo ${ }^{\mathrm{j}}$, A. O'Murchadha ${ }^{\mathrm{ab}}$, M. Ono ${ }^{\circ}$, S. Panknin ${ }^{k}$, L. Paul ${ }^{\mathrm{a}}$, C. Pérez de los Heros ${ }^{\text {ak }}$, J. Petrovic ${ }^{\mathrm{m}}$, A. Piegsa ${ }^{\text {ac }}$, D. Pieloth ${ }^{\mathrm{t}}$, R. Porrata ${ }^{\mathrm{g}}$, J. Posselt ${ }^{\mathrm{al}}$, P. B. Price ${ }^{\mathrm{g}}$, G. T. Przybylski ${ }^{\mathrm{h}}$, K. Rawlins ${ }^{\mathrm{c}}$, P. Redl ${ }^{\mathrm{q}}$, E. Resconi $^{\mathrm{w}}, \mathrm{W}$. Rhode ${ }^{\mathrm{t}}$, M. Ribordy ${ }^{y}$, A. Rizzo $^{n}$, J. P. Rodrigues ${ }^{\mathrm{ab}}$, P. Roth $^{\mathrm{q}}$, F. Rothmaier ${ }^{\mathrm{ac}}$, C. Rott ${ }^{r}$, T. Ruhe ${ }^{t}$, D. Rutledge ${ }^{a j}$, B. Ruzybayev ${ }^{\mathrm{ae}}$, D. Ryckbosch ${ }^{\mathrm{v}}$, H.-G. Sander ${ }^{\text {ac }}$, M. Santander ${ }^{\text {ab }}$, S. Sarkar ${ }^{\text {af }}$, K. Schatto ${ }^{\text {ac }}$, T. Schmidt ${ }^{\mathrm{q}}$, A. Schönwald ${ }^{\mathrm{am}}$, A. Schukraft ${ }^{\mathrm{a}}$, A. Schultes ${ }^{\mathrm{al}}$, O. Schulz ${ }^{\mathrm{w}}$, M. Schunck ${ }^{\mathrm{a}}$, D. Seckel ${ }^{\text {ae }}$, B. Semburg ${ }^{\text {al }}$, S. H. Seo ${ }^{\text {ah }}$, Y. Sestayow ${ }^{\text {}}$, S. Seunarine ${ }^{\text {, }}$ A. Silvestri ${ }^{\mathrm{x}}$, A. Slipak ${ }^{\mathrm{aj}}$, G. M. Spiczak ${ }^{\mathrm{ag}}$, C. Spiering ${ }^{\mathrm{am}}$, M. Stamatikos ${ }^{\mathrm{r}, 2}$, T. Stanev ${ }^{\text {ae }}$, G. Stephens ${ }^{\text {aj }}$, T. Stezelberger ${ }^{\text {h }}$ R. G. Stokstad ${ }^{\text {h }}$, A. Stössl ${ }^{\text {am }}$, S. Stoyanov ${ }^{\text {ae }}$, E. A. Strahler ${ }^{n}$, T. Straszheim ${ }^{q}$, M. Stür ${ }^{k}$, G. W. Sullivan ${ }^{\text {, }}$, Q. Swillens ${ }^{\mathrm{m}}$, H. Taavola ${ }^{\text {ak }}$, I. Taboada ${ }^{\mathrm{e}}$, A. Tamburro ${ }^{\mathrm{ag}}$, A. Tepe ${ }^{\mathrm{e}}$,

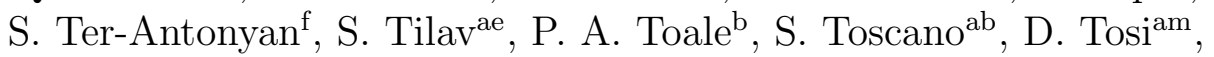
D. Turčan ${ }^{\mathrm{q}}$, N. van Eijndhoven ${ }^{\mathrm{n}}$, J. Vandenbroucke ${ }^{\mathrm{g}}$, A. Van Overloop ${ }^{\mathrm{v}}$, J. van Santen ${ }^{\mathrm{ab}}$, M. Vehring ${ }^{\mathrm{a}}$, M. Voge ${ }^{\mathrm{k}}$, C. Walck ${ }^{\mathrm{ah}}$, T. Waldenmaier ${ }^{\mathrm{i}}$, M. Wallraffa ${ }^{a}$ M. Walter ${ }^{\mathrm{am}}$, Ch. Weaver ${ }^{\mathrm{ab}}, \mathrm{C}$. Wendt ${ }^{\mathrm{ab}}$, S. Westerhoff ${ }^{\mathrm{ab}}$, N. Whitehorn ${ }^{\mathrm{ab}}$, K. Wiebe ${ }^{\mathrm{ac}}$, C. H. Wiebusch ${ }^{\mathrm{a}}$, D. R. Williams ${ }^{\mathrm{b}}$, R. Wischnewski ${ }^{\mathrm{am}}$, H. Wissing ${ }^{\mathrm{q}}$, M. Wolf ${ }^{\mathrm{w}}$, T. R. Wood ${ }^{\mathrm{u}}$, K. Woschnaggg, C. Xu ${ }^{\text {ae }}$, X. W. Xu ${ }^{\text {f }}$, G. Yodh ${ }^{\mathrm{x}}$, S. Yoshida ${ }^{o}$, P. Zarzhitsky ${ }^{\mathrm{b}}$, M. Zoll ${ }^{\text {ah }}$

${ }^{a}$ III. Physikalisches Institut, RWTH Aachen University, D-52056 Aachen, Germany

${ }^{b}$ Dept. of Physics and Astronomy, University of Alabama, Tuscaloosa, AL 35487, USA

${ }^{c}$ Dept. of Physics and Astronomy, University of Alaska Anchorage, 3211 Providence Dr., Anchorage, AK 99508, USA

${ }^{d}$ CTSPS, Clark-Atlanta University, Atlanta, GA 30314, USA

${ }^{e}$ School of Physics and Center for Relativistic Astrophysics, Georgia Institute of Technology, Atlanta, GA 30332, USA

${ }^{f}$ Dept. of Physics, Southern University, Baton Rouge, LA 70813, USA

${ }^{g}$ Dept. of Physics, University of California, Berkeley, CA 94720, USA

${ }^{h}$ Lawrence Berkeley National Laboratory, Berkeley, CA 94720, USA

${ }^{i}$ Institut für Physik, Humboldt-Universität zu Berlin, D-12489 Berlin, Germany

${ }^{j}$ Fakultät für Physik \&3 Astronomie, Ruhr-Universität Bochum, D-44780 Bochum,

Germany 
${ }^{k}$ Physikalisches Institut, Universität Bonn, Nussallee 12, D-53115 Bonn, Germany

${ }^{l}$ Dept. of Physics, University of the West Indies, Cave Hill Campus, Bridgetown BB11000, Barbados

${ }^{m}$ Université Libre de Bruxelles, Science Faculty CP230, B-1050 Brussels, Belgium

${ }^{n}$ Vrije Universiteit Brussel, Dienst ELEM, B-1050 Brussels, Belgium

${ }^{\circ}$ Dept. of Physics, Chiba University, Chiba 263-8522, Japan

${ }^{p}$ Dept. of Physics and Astronomy, University of Canterbury, Private Bag 4800, Christchurch, New Zealand

${ }^{q}$ Dept. of Physics, University of Maryland, College Park, MD 20742, USA

${ }^{r}$ Dept. of Physics and Center for Cosmology and Astro-Particle Physics, Ohio State University, Columbus, $\mathrm{OH} 43210$, USA

${ }^{s}$ Dept. of Astronomy, Ohio State University, Columbus, OH 43210, USA

${ }^{t}$ Dept. of Physics, TU Dortmund University, D-44221 Dortmund, Germany

${ }^{u}$ Dept. of Physics, University of Alberta, Edmonton, Alberta, Canada T6G 2 G7

${ }^{v}$ Dept. of Physics and Astronomy, University of Gent, B-9000 Gent, Belgium

${ }^{w}$ Max-Planck-Institut für Kernphysik, D-6917ry Heidelberg, Germany

${ }^{x}$ Dept. of Physics and Astronomy, University of California, Irvine, CA 92697, USA

${ }^{y}$ Laboratory for High Energy Physics, École Polytechnique Fédérale, CH-1015 Lausanne, Switzerland

${ }^{z}$ Dept. of Physics and Astronomy, University of Kansas, Lawrence, KS 66045, USA

${ }^{a a}$ Dept. of Astronomy, University of Wisconsin, Madison, WI 53706, USA

${ }^{a b}$ Dept. of Physics, University of Wisconsin, Madison, WI 53706, USA

${ }^{a c}$ Institute of Physics, University of Mainz, Staudinger Weg 7, D-55099 Mainz, Germany

${ }^{a d}$ Université de Mons, 7000 Mons, Belgium

${ }^{a e}$ Bartol Research Institute and Department of Physics and Astronomy, University of Delaware, Newark, DE 19716, USA

${ }^{a f}$ Dept. of Physics, University of Oxford, 1 Keble Road, Oxford OX1 3NP, UK

${ }^{a g}$ Dept. of Physics, University of Wisconsin, River Falls, WI 54022, USA

${ }^{a h}$ Oskar Klein Centre and Dept. of Physics, Stockholm University, SE-10691 Stockholm, Sweden

${ }^{a i}$ Dept. of Astronomy and Astrophysics, Pennsylvania State University, University Park, PA 16802, USA

${ }^{a j}$ Dept. of Physics, Pennsylvania State University, University Park, PA 16802, USA

${ }^{a k}$ Dept. of Physics and Astronomy, Uppsala University, Box 516, S-75120 Uppsala,

Sweden

${ }^{a l}$ Dept. of Physics, University of Wuppertal, D-42119 Wuppertal, Germany ${ }^{a m}$ DESY, D-15735 Zeuthen, Germany

\section{Abstract}

We present the results for a search of high-energy muon neutrinos with the IceCube detector in coincidence with the Crab nebula flare reported on 
September 2010 by various experiments. Due to the unusual flaring state of the otherwise steady source we performed a prompt analysis of the 79-string configuration data to search for neutrinos that might be emitted along with the observed $\gamma$-rays. We performed two different and complementary data selections of neutrino events in the time window of 10 days around the flare. One event selection is optimized for discovery of $E_{\nu}^{-2}$ neutrino spectrum typical of $1^{\text {st }}$ order Fermi acceleration. A similar event selection has also been applied to the 40-string data to derive the time-integrated limits to the neutrino emission from the Crab [35]. The other event selection was optimized for discovery of neutrino spectra with softer spectral index and $\mathrm{TeV}$ energy cut-offs as observed for various galactic sources in $\gamma$-rays. The $90 \% \mathrm{CL}$ best upper limits on the Crab flux during the 10 day flare are $4.73 \times 10^{-11} \mathrm{~cm}^{-2}$ $\mathrm{s}^{-1} \mathrm{TeV}^{-1}$ for an $E_{\nu}^{-2}$ neutrino spectrum and $2.50 \times 10^{-10} \mathrm{~cm}^{-2} \mathrm{~s}^{-1} \mathrm{TeV}^{-1}$ for a softer neutrino spectra of $E_{\nu}^{-2.7}$, as indicated by Fermi measurements during the flare. IceCube has also set a time-integrated limit on the neutrino emission of the Crab using 375.5 days of livetime of the 40-string configuration data. This limit is compared to existing models of neutrino production from the Crab and its impact on astrophysical parameters is discussed. The most optimistic predictions of some models are already rejected by the IceCube neutrino telescope with more than $90 \%$ CL.

Keywords: Neutrino and gamma flare, pulsar, nebula.

\section{Introduction}

The Crab supernova remnant, originating from a stellar explosion at a distance of $2 \mathrm{kpc}$ recorded in $1054 \mathrm{AD}$, consists of a central pulsar, a synchrotron nebula, and a surrounding cloud of expanding thermal ejecta [1]. Its bright and steady emission has made it a standard candle for telescope calibration. However, the photon emission stability in the X-ray and in the $\gamma$-ray regions is recently being questioned by a number of satellite experiments. As a matter of fact, a 7\% decline of the Crab flux in the $3-100 \mathrm{keV}$ region, larger at higher energies, has been observed in the period between 2008 and 2010 by the Fermi Gamma-ray Burst monitor and confirmed by Swift/BAT, RXTE/PCA, and INTEGRAL (IBIS) [2]. The pulsed emission from RXTE/PCA observations is consistent with the observed pulsar spindown suggesting that the decline is due to changes in the nebula and not in the pulsar. 
The source of energy that powers the Crab is the spin-down luminosity of the pulsar. The measured spin-down luminosity of the pulsar is $\sim 5 \times 10^{38} \mathrm{erg}$ $\mathrm{s}^{-1}$ and its rotational period is $33 \mathrm{~ms}$. While a small fraction of this energy goes into the pulsed emission, most of it is carried by a highly magnetized wind of relativistic plasma, the composition of which is not known. Both pure $e^{ \pm}$plasma models and a mixture of $e^{ \pm}$and protons or ions have been proposed $[1,[3,4,[6,10]$. The wind terminates in a standing shock and transfers some of the energy to accelerating particles. A part of this energy is converted into synchrotron emission from radio to $\mathrm{MeV} \gamma$-rays by a population of high energy electrons radiating in the nebular magnetic field. The observations of the synchrotron emission from the Crab up to the $\mathrm{MeV}$ energies, make the Crab an undisputed galactic accelerator able to inject electrons up to energies $\sim 10^{15} \mathrm{eV}$. These high energy electrons inevitably interact with the ambient photon fields through inverse Compton scattering, resulting in the production of high-energy $\gamma$-rays observable in the TeV regime [13, 14, 15]. The synchrotron emission from the Crab has an integrated luminosity of $\sim 1.3 \times 10^{38} \mathrm{erg} \mathrm{s}^{-1}$, that is, at least $\sim 26 \%$ of the spin-down luminosity of the pulsar is involved in the acceleration of electrons in the energy range $10^{11}-10^{15} \mathrm{eV}[1]$. On the other hand, the presence of hadrons in the pulsar wind and the amount of energy transported by them remain as some of the unresolved and interesting questions about the Crab Nebula and plerions in general.

Protons and ions do not lose their energy as efficiently as electrons, and hence it is more difficult to observe the products of their interactions. The dominant processes, discussed below, are proton-proton and proton- $\gamma$ interactions, and both processes generate $\gamma$-rays and neutrinos through meson decays. Hence, neutrinos constitute an unique signature for hadron acceleration while hadronic $\gamma$-ray production has to be disentangled from inverse Compton emission. Hadronic models of the Crab emission assume that the pulsar wind is composed of a mixture of electrons and ions. These models predict that a significant part of the rotational energy lost by the pulsar is transferred through the shock radius to relativistic nuclei in the pulsar wind. Relativistic nuclei injected into the nebula can interact with the nebula matter, and produce cosmic rays and neutrinos via pion decay. Neutrino production by protons and nuclei interacting in the pulsar wind in the Crab have been discussed in Ref. [3, 4]. According to these models, the nuclei can generate Alfvén waves just above the pulsar wind shock. These Alfvén waves will resonantly scatter off and accelerate the positrons and electrons 
that create the synchrotron emission. In the model described in Ref. [6] neutrinos are produced by heavy nuclei accelerated by the rotating neutron star that photo-disintegrate in collisions with soft photons. These models predict between $1-5$ events per year in a cubic-kilometer detector such as IceCube when accounting for neutrino oscillations. Inelastic nuclear collisions are considered in Ref. [3]. In this paper the predicted rates depend on the Lorentz factor, $\Gamma$, of nuclei injected by the pulsar and the effective target density. The thermal matter distribution in the Crab is far from being uniform but forms filaments. For relativistic protons the effective target density is also affected by the structure of the magnetic field in and around these filaments. The authors in Ref. [3] provide several expected neutrino fluxes from the Crab Nebula as a function of energy, for different assumptions on these two parameters. For the highest values of the effective target density, IceCube begins to have the sensitivity to probe the highest possible values around $\Gamma \lesssim 10^{7}$ while the favored values of the upstream Lorentz factor of the wind are $\Gamma \sim 10^{6}$ [5] .

Acceleration of positive ions near the surface of a young rotating neutron star $\left(\lesssim 10^{5}\right.$ yrs) has also been investigated in Ref. [7]. This model describes how positive ions can be accelerated to $\sim 1 \mathrm{PeV}$ in rapidly-rotating pulsars, with typical magnetic fields $\left(B \sim 10^{12} \mathrm{G}\right)$, by a potential drop across the magnetic field lines of the pulsar. Assuming that the star's magnetic moment $\mu$ and the angular velocity $\Omega$ satisfy the relation $\vec{\mu} \cdot \vec{\Omega}<0$, protons are accelerated away from the stellar surface. Beamed neutrinos (in coincidence with the radio beam) are produced by such high energy protons interacting with the star's radiation field when the $\Delta$ production threshold is surpassed. Observation of these neutrinos could validate the existence of a hadronic component and a strong magnetic field near the stellar surface that accelerates the charged particles. The predictions in Ref. [8] based on this model account for $\sim 45$ neutrino events/yr from the Crab in a cubic-kilometer detector in the most optimistic scenario where the fraction of charge depletion is assumed to be $f_{d} \sim 1 / 2$. In this paper we will show that IceCube data severely constrains these optimistic predictions of the model.

In Ref. [12] a mean prediction of 1.2 neutrino events per year for $E_{\nu}>$ $1 \mathrm{TeV}$ was calculated for an underwater cubic-kilometer detector. This prediction is based on the H.E.S.S. measured $\gamma$-ray spectrum [13] assuming that all the $\gamma$-rays observed by H.E.S.S. up to $40 \mathrm{TeV}$ are produced by pion decay and that the absorption of $\gamma$-rays is negligible. A similar calculation connecting photon and neutrino fluxes was done in Ref. [9] predicting about 5 
events from the Crab accounting for neutrino oscillations. For a summary of some of the models on neutrino spectra the reader is referred to [10].

From Sep. 19 to 22, 2010 the AGILE satellite [16, 17] reported an enhanced $\gamma$-ray emission above $100 \mathrm{MeV}$ from the Crab nebula. The flare, however, was not detected in X-rays by INTEGRAL [20] observations between Sep. 12 and 19 partially overlapping with AGILE observations. It was also not confirmed by the SWIFT/BAT [21] in the 15-150 keV range nor by RXTE [22] on a dedicated observation of the Crab on Sep. 24. The observation was later confirmed by the Large Area Telescope on board of the Fermi Gamma-Ray Space Telescope that detected a flare of $\gamma$-rays $\left(E_{\gamma}>100 \mathrm{MeV}\right)$ with a duration of $\sim 4$ days between Sep. 19-22 in the Crab direction [24]. The observed energy spectrum during the flare interval was consistent with a negative power-law with a spectral index of $-2.7 \pm 0.2$. The flux increase was a factor $5.5 \pm 0.8$ above the average flux from the Crab. Fermi also detected another flare of 16 days in Feb. 2009 corresponding to a flux increase of a factor $3.8 \pm 0.5$ but much softer spectral index $(-4.3 \pm 0.3)$. The ARGOYBJ collaboration also issued an ATel on Sep. 2010 on the observation of an enhancement of the $\mathrm{TeV}$ emission for the same period of time but with a wider interval of 10 days. The enhanced $\mathrm{TeV}$ emission corresponded to a flux about 3-4 times higher than the usual Crab flux in TeV energies [23]. However, this observation was not confirmed by MAGIC [25] nor VERITAS [26]; Imaging Cherenkov Telescopes in a similar energy range as ARGO-YBJ. The spectral and timing properties of the flares indicate that the $\gamma$-rays are emitted via synchrotron radiation from $\mathrm{PeV}$ electrons from a region smaller than $1.4 \times 10^{-2}$ pc. This dimension is comparable to the jet knots observed close to the termination shock of the Crab Nebula [19]. Even though the Crab has always been considered to be a source of synchrotron emission, the flare represents a challenge to shock diffusive acceleration theory [24]. Nonetheless, explanations of the high variability due to electromagnetic phenomena have been proposed in Ref. [11] where the emission comes from a part of the pulsar wind shock 3 .

The unusual flaring state of this otherwise steady source, the intensity of

\footnotetext{
${ }^{3}$ During the final stage of the editing of this paper another large flare was observed from the Crab [18]. This flare is even more intense than the one observed in September and is being studied by various experiments. Hence, IceCube analysis will happen when results from Fermi, other X-ray satellites and other $\mathrm{TeV}$ ground based experiments will be available.
} 
the flare, and the experimental observations in $\gamma$-rays motivated this search for neutrinos in IceCube in coincidence with the Crab flare of Sep. 2010. The IceCube collaboration started a prompt analysis of the then-running 79string configuration. The time window selected for this analysis was the 10 days interval reported by ARGO-YBJ from September 17 to September 27, which contains the Fermi flare window. An unbinned maximum likelihood (LLH) method described in Ref. [27] has been applied to search for an excess of neutrinos in coincidence with the enhanced $\gamma$-ray emission from the Crab. The non observation of neutrinos would reinforce pure electromagnetic emission scenarios and determine the level at which hadronic phenomena superimposed on an electromagnetic scenario can be probed.

The IceCube Neutrino Observatory is a neutrino telescope installed in the deep ice at the geographic South Pole. The final configuration comprises 5,160 photomultipliers (PMTs) [29] along 86 strings instrumented between $1.5-2.5 \mathrm{~km}$ in the ice. Its design is optimized for the detection of high energy astrophysical neutrinos with energies above $\sim 100 \mathrm{GeV}$. The observation of cosmic neutrinos will be a direct proof of hadronic particle acceleration and will reveal the origins of cosmic rays $(\mathrm{CR})$ and the possible connection to shock acceleration in Supernova Remnants (SNR), Active Galactic Nuclei (AGN) or Gamma Ray Bursts (GRBs). The IceCube detector uses the Antarctic ice as the detection volume where muon neutrino interactions produce muons that induce Cherenkov light. The light propagates through the transparent medium and can be collected by PMTs housed inside Digital Optical Modules (DOMs). The DOMs are spherical, pressure resistant glass vessels each containing a $25 \mathrm{~cm}$ diameter Hamamatsu photomultiplier and its associated electronics. Eight densely instrumented strings equipped with higher quantum efficiency DOMs form, together with 12 adjacent IceCube strings, the DeepCore array that increases the sensitivity for low energy neutrinos down to about $10 \mathrm{GeV}$. Detector construction finished during the austral summer of 2010-11.

This paper describes in Sec. 2 the data selection, the comparison to simulation, and the detector effective area and angular resolution for this search; in Sec. 3 we summarize the analysis method used; in Sec. 4 the results for the flare search are presented. Given the null result, upper limits are provided. In Sec. 5 the time-integrated upper limits based on 1 year of data of the 40-string configuration are presented to summarize what is the impact of the IceCube most sensitive limit on existing neutrino production models for the Crab. Conclusions are given in Sec. 6. 


\section{Data Selection and Comparison to Monte Carlo}

The detection principle of IceCube is based on the charge and time measurement of the Cherenkov photons induced by relativistic charged particles passing through the ice sheet. The PMT signal is digitized with dedicated electronics included in the DOMs [31]. A DOM is triggered when the PMT voltage crosses a discriminator threshold set at a voltage corresponding to about 1/4 photoelectron. Various triggers are used in IceCube. The results shown here are based on a simple multiplicity trigger requiring that the sum of all triggered DOMs in a rolling time window of $5 \mu \mathrm{s}$ is above 8 (SMT8). The duration of the trigger is the amount of time that this counter stays at or above 8 as the time window keeps moving. Once the trigger condition is met, all local coincidence hits are recorded in a readout window of $\pm 10 \mu$ s for the 40-string run and of ${ }_{-4}^{+6} \mu \mathrm{s}$ (to reduce the noise rate) in the 79-string run. IceCube triggers primarily on down-going muons at a rate of about $1.8 \mathrm{kHz}$ in the 79-string configuration. Variation in the trigger rate determined by atmospheric muons is about $\pm 10 \%$ due to seasonal changes [32]. Seasonal variations in atmospheric neutrino rates are expected to be a maximum of $\pm 4 \%$ for neutrinos originating near the polar regions. Near the equator, atmospheric variations are much smaller and the variation in the number of events is expected to be less than $\pm 0.5 \%$ [33].

For searches of neutrino point sources in the northern sky, IceCube can use the Earth as a shield to reduce the background of atmospheric muons and detect up-going muons induced by neutrinos. In the northern sky these searches are sensitive to neutrinos in the $\mathrm{TeV}-\mathrm{PeV}$ region.

In order to reconstruct muon tracks a LLH-based reconstruction is performed at the South Pole (L1 filter) providing a first order background rejection of poorly reconstructed events and a selection of high energy muons for the southern sky. The data sent through the satellite to the North undergo further processing that includes a broader range of more CPU consuming reconstructions. This offline processing also provides useful variables for background rejection, measurements of the energy and of the angular uncertainty, and selects about $35 \mathrm{~Hz}$ of the SMT8 data. However, the offline processing requires a fair amount of time to be finalized and is not suitable for expedited analysis. For the analysis of the Crab flare we used a dedicated selection for target of opportunity programs [36]. This online event selection and reconstruction is called the online Level 2 filter and selects about $4 \mathrm{~Hz}$ of data. It provides a reduced data rate (compared to the standard online 
data) because of stricter cuts than in the offline filter. The loss of sensitivity of this stream of data is marginal for $E^{-2}$ neutrino spectra.

The online L2 filter performs a 8-fold iterative single photoelectron (SPE) LLH fit for events with the number of DOMs triggered fewer than 300 and a 4-fold iterative SPE fit otherwise. These SPE fits are seeded by a track obtained using a single iteration LLH fit [34]. While the online Level 2 selects good quality tracks and high energy muons from the northern sky, it is dominated by the background of down-going atmospheric muons and therefore further cuts have to be applied before performing neutrino source searches. Experimental and simulated data are processed and filtered in the same way. The data used for this search concern the period from 2010/08/10 to $2010 / 10 / 12$. In this period the detector was running in a stable configuration. The total live time for that period (considering deadtimes) is 60.9 days. Figure 1 shows the data rate of each run included during the selected time window as well as the South Pole atmospheric temperature. As can be seen at this level, the rate is dominated by down-going atmospheric muons, which display larger weather-dependent variations than the final up-going neutrino events.

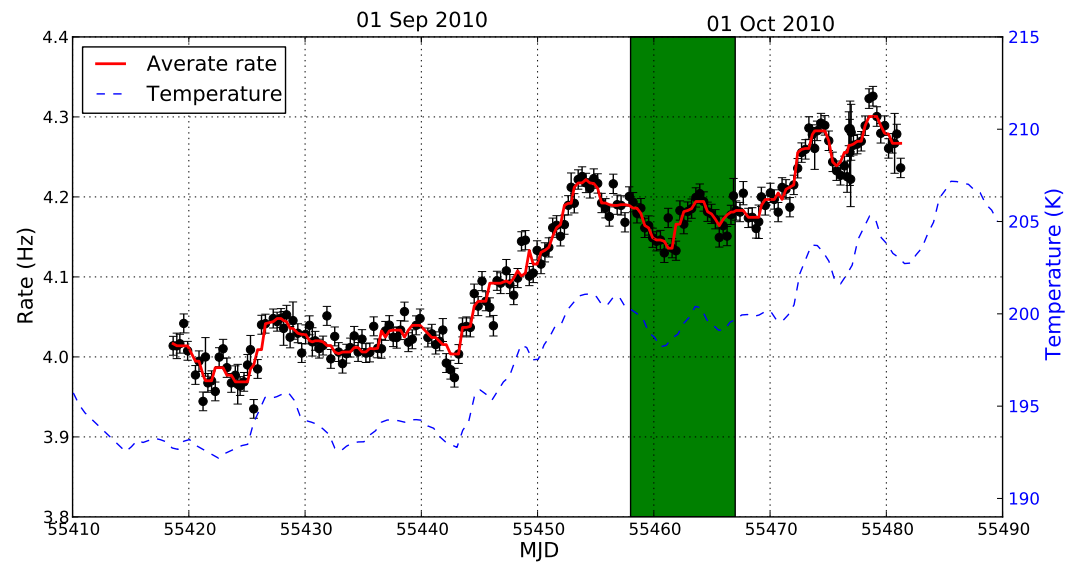

Figure 1: Data rates per run of the online Level 2 filter of 79 IceCube strings in the time window of the Crab flare. The green bar indicates the flaring interval used in this analysis according to ARGO measurements [23]. The blue dotted line indicates the temperature in the middle stratosphere of the South Pole according to [32].

We have performed two dedicated selections starting from the online L2 filter that we describe below. 


\subsection{Straight Cuts Data Selection}

This dataset is obtained by requiring a good level of reconstruction and ensuring degree level accuracy in the tracking errors to reject the misreconstructed down-going atmospheric muons from the real up-going atmospheric neutrino sample. The variables used are determined in the offline data processing and have been used for the 40-string point-source analyses in [35] and [28]. The final cut level can be achieved by applying the following series of cuts on a number of variables to obtain a good agreement between data and the simulation of atmospheric neutrinos, with a contamination of the order of $5 \%$ of atmospheric muons, mainly muons from two cosmic ray showers in coincidence in the same readout window. Having these muons with different directions gives hit patterns that confuse the reconstruction so that at times the result is a misreconstructed up-going track. The cuts are:

$$
N_{d i r} \geq 5 ; L_{d i r}>200 m ; \sigma_{c r}<5^{\circ} ; L_{r e d} \leq\left\{\begin{array}{l}
7.4 \text { if } L_{r e d}^{\prime} \leq 6.4 \\
8.0 \text { otherwise }
\end{array}\right.
$$

where:

- $\mathbf{N}_{\text {dir }}$ : is the number photons detected within -15 and 75 ns with respect to the expected arrival time of unscattered photons from the reconstructed muon-track. Scattering of photons in the ice causes a loss of directional information and will delay them with respect to the unscattered expectation;

- $\mathbf{L}_{\text {dir }}$ : is the maximum distance in meters between direct photons projected along the best muon track solution;

- $\boldsymbol{\sigma}_{c r}$ : is the uncertainty on the reconstructed track direction given by the LLH-based track reconstruction estimated by a method based on the Cramer-Rao inequality [37]; and

- $\mathbf{L}_{\text {red }}$ and $\mathbf{L}_{\text {red }}^{\prime}$ : are the standard reduced and modified LLH values respectively. The reduced LLH is defined as the $-\log _{10}$ of the LLH value of the track reconstruction divided by the number of degrees of freedom. The number of degrees of freedom is the number of hit DOMs minus five fit parameters, two angles and three coordinates of a reference point along the track. It was found by comparing background rejection efficiency to signal selection efficiency that a good variable for 
rejection of background for low energy events is the number of hit DOMs minus an effective number of degrees of freedom of 2.5 .

An additional cut to select events in the direction of the Crab $\left(\Theta_{\mathrm{Crab}}=\right.$ $122^{\circ}$ at the South Pole) has also been applied: $\Theta_{\text {Crab }}-10^{\circ}<\theta_{\text {rec }}<\Theta_{\text {Crab }}+$ $10^{\circ}$, where $\theta_{\text {rec }}$ is the reconstructed zenith angle of the muon track. No further selection in right ascension has been applied. In Tab. 1 the selected number of events and the expected number of atmospheric neutrinos and muons are given. The final number of events selected for the 10 day window of the flare is 354 .

\subsection{BDT Data Selection}

The second dataset is obtained by using a multi-variate learning machine. In particular this data selection is based on the knowledge and experience from previous analyses looking for solar Weakly Interactive Massive Particles (WIMPs) with the IceCube detector [38]. During the austral winter the Sun is below the horizon at the South Pole and its maximum declination is equal to the obliquity of the ecliptic, $23.4^{\circ}$. Since the Crab Nebula lies fairly close to the ecliptic plane, the strategies and cuts that are optimized for this specific direction can be applied for the Crab direction.

Starting with the online L2 filtered data selection, as described above, a number of additional cuts were applied. The hereby selected events fulfill criteria of horizontal tracks passing the detector, to further minimize vertical tracks associated with background events. Additionally, the cuts were chosen to reduce the tails of distributions of the background into the signal region:

$z_{\text {travel }}>-10 \mathrm{~m} ; \sigma_{C O G z}<170 \mathrm{~m} ; \sigma_{c r}<10^{\circ} ; \rho_{a v}<150 \mathrm{~m} ; t_{\text {accu }}<3000 \mathrm{~ns}$

where:

- $\boldsymbol{z}_{\text {travel }}$ : measures the difference in the $z$ positions of the center of gravity (COG) of the hits at the beginning of an event (first $1 / 4$ of the hits in time) and the COG at the end of the event (last $3 / 4$ of the hits in time);

- $\boldsymbol{\sigma}_{C O G_{z}}$ : is the uncertainty in meters of the $z$-coordinate of the COG;

- $\boldsymbol{\rho}_{a v e}$ : is the mean minimal distance between the LLH track and the hit DOMs; and 
- $\boldsymbol{t}_{\text {accum }}$ : is the accumulation time, defined as the time until $75 \%$ of the total charge develops in ns.

Boosted Decision Trees (BDTs) [39], multi-variate learning machines, were used in the final analysis step to classify events as signal-like or background-like. Eleven event observables, split in two sets of 5 and 6 each, were obtained by choosing parameters with low correlation in background (correlation coefficient $|c|<0.5$ ), but high discriminating power between signal and background. The selected observables include $N_{d i r}, L_{d i r}, \sigma_{c r}$ and $L_{r e d}^{\prime}$ as described within the straight cuts data selection in Sec.2.1 and $z_{\text {travel }}$ from above. Additionally, observables specifying the geometry, the time evolution of the hit pattern, the quality and consistency of the various track reconstructions that is defined through the opening angle between the line-fit and the LLH tracks, and the number of hit strings are used. Training was done with simulated signal events for a soft neutrino spectrum of $E^{-3}$ that also well represents the case of an $E^{-2}$ spectrum with a TeV cut-off. A set of off-time real data, not used in the flare analysis, was used for training as background. The final sample is defined by a cut on the combined output (score) of the two BDTs. As in the case of the straight cuts sample, an additional requirement of reconstructed zenith tracks within $\pm 10^{\circ}$ from the Crab has been applied. In Tab. 1 the selected number of events and the expected number of atmospheric neutrinos and muons are given. The final number of events selected for the 10 day window of the flare is 660 events in the northern sky.

\begin{tabular}{c|c|c|c|c}
\hline Cut Level & $\begin{array}{c}\text { Data rate } \\
(\mathrm{Hz})\end{array}$ & $\begin{array}{c}\text { Atm. } \mu \text { rate } \\
(\mathrm{Hz})\end{array}$ & $\begin{array}{c}\text { Atm. } \nu_{\mu} \text { rate } \\
(\mathrm{Hz})\end{array}$ & $\begin{array}{c}\mathrm{E}^{-2} \text { Eff. } \\
(\%)\end{array}$ \\
\hline Trigger & 1,800 & 1,800 & $2.59 \times 10^{-2}$ & - \\
Online Level 2 & 4.03 & 3.11 & $7.2 \times 10^{-3}$ & 100 \\
Straight Cuts & $4.6 \times 10^{-4}$ & $\sim 0$ & $4.8 \times 10^{-4}$ & 55 \\
BDT & $8.4 \times 10^{-4}$ & $\sim 0$ & $8.2 \times 10^{-4}$ & 61 \\
\hline
\end{tabular}

Table 1: Data, atmospheric muon, and neutrino expected background rates for different cut progression. The signal efficiency for an $E^{-2}$ neutrino spectrum assuming an emission $\pm 10^{\circ}$ around the Crab with respect to the online Level 2 is also shown. 


\subsection{Comparison Data-Monte Carlo and Detector Performance}

The simulation of atmospheric and signal neutrinos that is used for determining the selection efficiency, the performance of the detector and to calculate upper limits is based on the neutrino generator ANIS [40] and the deep inelastic neutrino-nucleon cross sections with CTEQ5 parton distribution functions [41]. Neutrino simulation can be weighted for different fluxes, accounting for the probability of each event to occur. In this way, the same simulation sample can be used to represent atmospheric neutrino models such as Bartol [42] and Honda [43] neutrino fluxes from pion and kaon decays (conventional flux) and a variety of models for the charm component (prompt flux) [44, 45]. Muons from CR air showers were simulated with CORSIKA [46] with the SIBYLL hadronic interaction models [47]. An October polar atmosphere, an average case over the year, is used for the CORSIKA simulation. Seasonal variations are therefore to be expected less than $\pm 10 \%$ in event rates [32]. Muon propagation through the Earth and ice are done using MMC [48]. This simulation is used to verify the level of agreement of data and $\mathrm{MC}$ from trigger level to Level 1 and to understand the level of contamination at final cut level. For the optical properties of the ice we used a model obtained from calibrations using the LEDs in the DOMs called flashers [49]. This model produces a better agreement between data and MC than the model previously used [50]. The simulation propagates the photon signal to each DOM using light tracking software described in [51]. The simulation of the DOMs includes their angular acceptance and electronics. The systematic errors on the simulation of the signal used to produce the upper limits have been evaluated and presented in Sec. 6 of Ref. [35] describing the 40-string time-integrated point source search. The main uncertainties on the limits for an $E^{-2}$ signal of muon neutrinos come from photon propagation, absolute DOM efficiency, and uncertainties in the Earth density profile and muon energy loss, accounting for a total of $16 \%$.

Figure 2 shows the data and simulation comparison for some variables at the final cut level for the two data samples. As can be seen, the BDT sample increases the overall rate by allowing more low quality reconstructed events (high $L_{r e d}$ ) than the straight cut sample. This is translated into a higher

neutrino effective area at low energies but also a worse angular resolution as can be seen in figure 3 . 

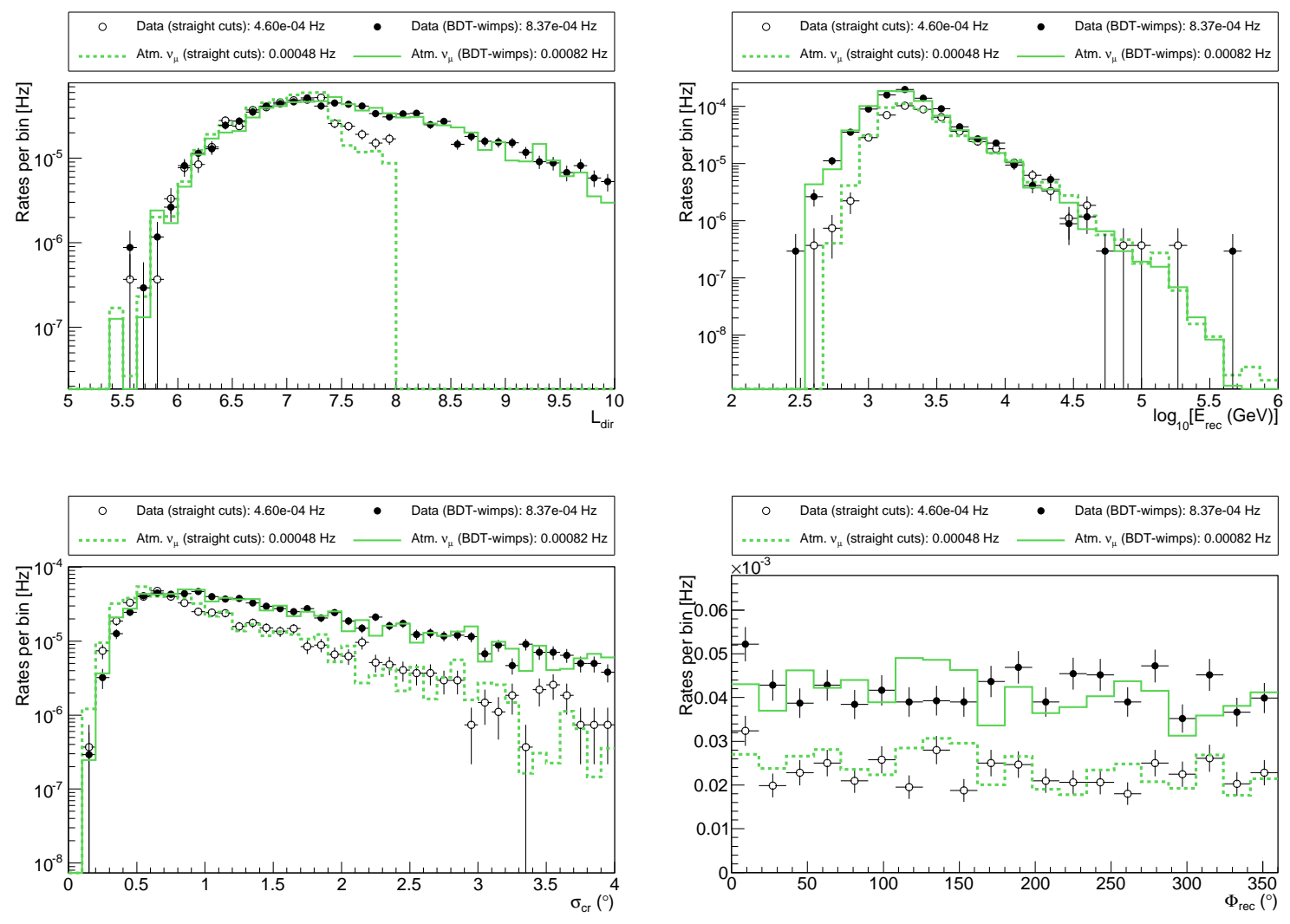

Figure 2: The top-left plot shows the reduced log-likelihood $\left(L_{r e d}\right)$, as defined in section 2 , distribution for both data (dots) and atmospheric neutrino simulation (green lines) for the two data samples. The distribution of the reconstructed energy is shown on the top-right plot. The estimated angular error given by the track reconstruction algorithm using the Cramer-Rao upper bound is shown on the bottom-left plot while the bottom-right shows the azimuth distribution of the final data samples. 

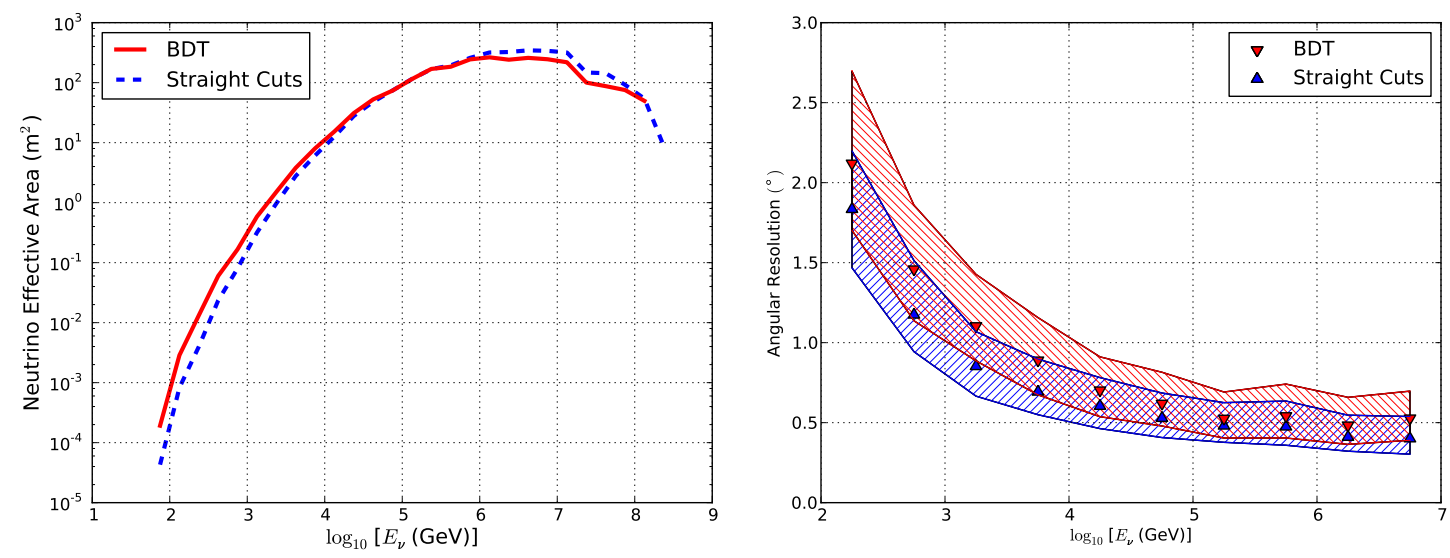

Figure 3: Left: Muon neutrino effective area for the two final data samples in a zenith bin of $\pm 10^{\circ}$ from the direction of the Crab Nebula. Right: Angular resolution defined as the median of the point spread function as a function of the neutrino energy for the two data samples. The shaded areas represent a $\pm 10 \%$ area of the point spread function.

\section{Likelihood analysis}

The method used for this analysis is an unbinned likelihood method [27]. This method looks for a localized statistically significant excess of neutrinos above the background in the direction of the Crab in coincidence with the flare. The same analysis technique has already been applied to AGN flare searches in IceCube [28]. The method uses both the reconstructed direction of the events as well as an energy proxy, the reconstructed visible muon energy, to discriminate any possible signal from background during the time interval of the flare. We consider the largest reported time window of 10 days by ARGO-YBJ. The applied method describes the data as a two component mixture of signal and background. For a data set with $\mathrm{N}$ total events the probability density of the $i^{\text {th }}$ event is given by:

$$
\frac{n_{s}}{N} \mathcal{S}_{i}+\left(1-\frac{n_{s}}{N}\right) \mathcal{B}_{i},
$$

where $\mathcal{S}_{i}$ is the density distribution for the signal hypothesis and $\mathcal{B}_{i}$ for background. The parameter $n_{s}$ is the number of signal events and one of the free parameters of the likelihood maximization together with the spectral index, $\gamma$, of the signal spectrum distribution. The likelihood of the data is 
the product of all event probability densities:

$$
\mathcal{L}\left(n_{s}, \gamma\right)=\prod_{i=1}^{N}\left[\frac{n_{s}}{N} \mathcal{S}_{i}+\left(1-\frac{n_{s}}{N}\right) \mathcal{B}_{i}\right]
$$

The likelihood is then maximized with respect to $n_{s}$ and $\gamma$, giving the best fit values $\hat{n}_{s}$ and $\hat{\gamma}$. The null hypothesis is given by $n_{s}=0(\gamma$ has no meaning when no signal is present). The likelihood ratio test-statistic is defined as:

$$
T S=-2 \log \left[\frac{\mathcal{L}\left(n_{s}=0\right)}{\mathcal{L}\left(\hat{n}_{s}, \hat{\gamma}_{s}\right)}\right] .
$$

The background probability distribution function, or pdf, $\mathcal{B}_{i}$, is given by:

$$
\mathcal{B}_{i}=\mathcal{B}_{i}^{\text {space }}\left(\theta_{i}, \phi_{i}\right) \mathcal{B}_{i}^{\text {energy }}\left(E_{i}, \theta_{i}\right) \mathcal{B}_{i}^{\text {time }}\left(t_{i}, \theta_{i}\right),
$$

and is computed using the distribution of data itself. The spatial term $\mathcal{B}_{i}^{\text {space }}\left(\theta_{i}, \phi_{i}\right)$ is the event density per unit solid angle as a function of the local coordinates. The energy probability, $\mathcal{B}_{i}^{\text {energy }}\left(E_{i}, \theta_{i}\right)$, is determined from the energy proxy distribution of data as a function of the cosine of the zenith angle, $\theta_{i}$. This energy proxy, described in detail in [35], uses the density of photons along the muon track due to stochastic energy losses of pair production, bremsstrahlung and photonuclear interactions which dominate over ionization losses for muons above $1 \mathrm{TeV}$. The time probability $\mathcal{B}_{i}^{\text {time }}\left(t_{i}, \theta_{i}\right)$ of the background can be taken to be flat for this case of a 10 day time interval ignoring the seasonal modulations.

The signal pdf $S_{i}$ is given by:

$$
\mathcal{S}_{i}=\mathcal{S}_{i}^{\text {space }}\left(\left|\vec{x}_{i}-\vec{x}_{s}\right|, \sigma_{i}\right) \mathcal{S}_{i}^{\text {energy }}\left(E_{i}, \theta_{i}, \gamma_{s}\right) \mathcal{S}_{i}^{\text {time }}\left(t_{i}\right)
$$

where $\mathcal{S}_{i}^{\text {space }}$ depends on the angular uncertainty of the event $\sigma_{i}$ and the angular difference between the event position $\vec{x}_{i}$ from the source position $\vec{x}_{s}$. The density function $\mathcal{S}_{i}^{\text {energy }}$ is a function of the reconstructed energy proxy $E_{i}$, and the spectrum $\gamma_{s}$ is calculated from an energy distribution of simulated signal in a zenith band that contains the source. The signal time probability, $\mathcal{S}_{i}^{\text {time }}$, depends on the particular signal hypothesis. In this analysis we adopt a simple cut in time between $t_{\min }$ and $t_{\max }$, which can be expressed as:

$$
\mathcal{S}_{i}^{\text {time }}=\frac{H\left(t_{\max }-t_{i}\right) \times H\left(t_{i}-t_{\min }\right)}{t_{\max }-t_{\min }}
$$


where $t_{i}$ is the arrival time of the event, $t_{\max }$ and $t_{\min }$ are the upper and lower bounds of the time window defining the flare, and $\mathrm{H}$ is the Heavyside step function.

The significance of the result is evaluated by comparing the test-statistic with a distribution obtained by performing the same analysis over a set of background-only scrambled data sets. The fraction of trials above the test-statistic value obtained from data is referred to as the $p$-value, with smaller $p$-values indicating that the background-only (i.e. null) hypothesis is increasingly disfavored compared to the signal-plus-background hypothesis as a description of the data. This leads to the definition of the discovery potential: the average number of signal events required to achieve a $p$-value less than $2.87 \times 10^{-7}$ (one-sided $5 \sigma$ ) in $50 \%$ of trials. Similarly, the sensitivity is defined as the average signal required to obtain, in $90 \%$ of trials, a teststatistic greater than the median test-statistic of background-only scrambled samples.

\section{Results}

The method described in section 3 has been applied to both data samples, the one obtained with straight cuts and the one obtained using the BDTs. In both cases the best fit resulted in $n_{s}=0$ (i.e. an under-fluctuation). Figure 4 shows the event distribution for those events with a $\frac{\mathcal{S}_{i}}{\mathcal{B}_{i}}>1$, that is, only events inside the flare window that contribute to the likelihood.

As can be seen, due to its higher neutrino efficiency at energies below $10 \mathrm{TeV}$ the BDT sample has more atmospheric neutrino events. Since the background estimation depends on the sample, the signal-to-background ratios are different for the same events in the two samples. The highest event weight comes from the straight cuts sample.

Table 2 shows the upper limits set by both data samples for different neutrino spectra. Each upper limit is shown both in terms of number of

signal events that can be rejected at $90 \% \mathrm{CL}, n_{s}^{90 \%}$, and the flux limit on muon neutrinos, $\Phi_{\nu_{\mu}}^{90 \%}$, for a 9.28 day interval in units of $\mathrm{cm}^{-2} \mathrm{~s}^{-1} \mathrm{TeV}^{-1}$, i.e. $\frac{d N^{90 \%}}{d E}=\Phi_{\nu_{\mu}}^{90 \%}\left(\frac{E}{\mathrm{TeV}}\right)^{-\gamma}$.

The analysis described and the results given in Tab. 2 rely on the fact that background simulation can be performed by scrambling the right ascension in real data (even if a signal is present in the data sample the scrambling will dilute it over the background). This method of estimating the background 

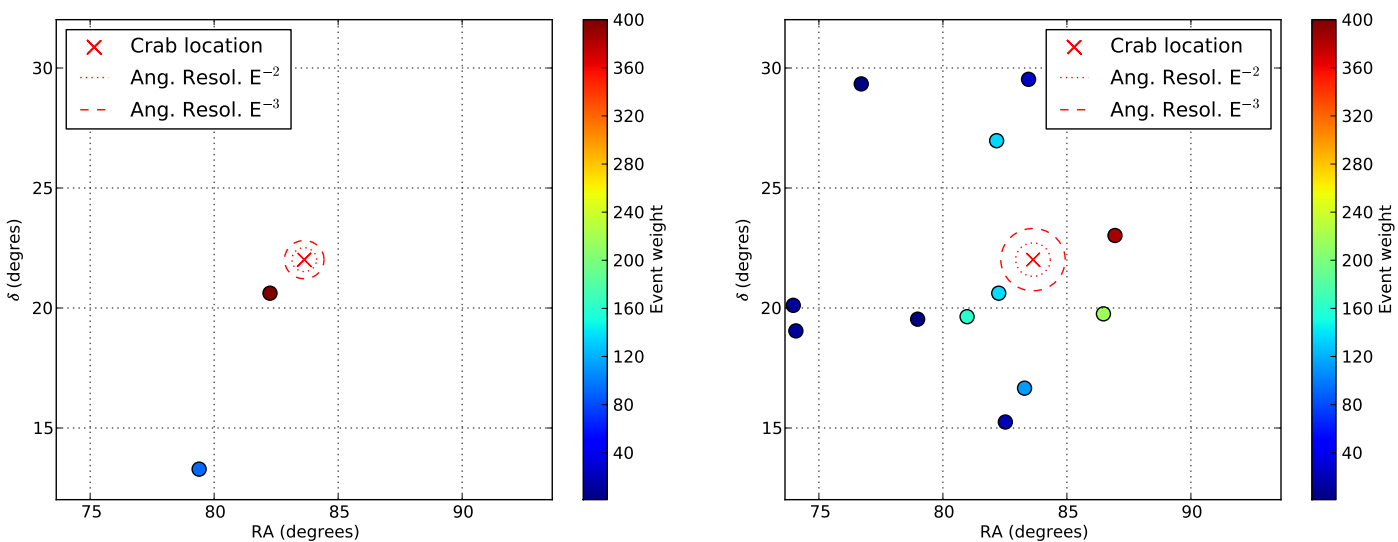

Figure 4: Left: Event distribution for the straight cuts for events with $\frac{\mathcal{S}_{i}}{\mathcal{B}_{i}}>1$. The color code represents the event signal-over-background ratio. Right: Same distribution for the BDT sample.

gives robust $p$-values in terms of systematic uncertainties. Systematic uncertainties only affect the estimate of the signal flux from the source and the upper limits. The systematic uncertainties on the expected flux come from photon propagation in ice, absolute DOM sensitivity $( \pm 8 \%)$, and uncertainties in the Earth density profile as well as muon energy loss. The main uncertainty however is the modeling of Antarctic ice and its effect on the photon propagation. In IceCube different ice models have been devised. The variation in the upper limits depending on the photon propagation model used are within $<10 \%$. Overall the uncertainty on upper limits is $16 \%$.

\section{Impact of IceCube time-integrated limits on models from the Crab}

The main goal of the IceCube telescope is the search for cosmic neutrino signals that might explain the astrophysical phenomena that give rise to the cosmic ray emission. In the absence of detection, constraining models can also provide insights about the nature of these phenomena. The best available neutrino flux limits for the Crab are based on the time-integrated analysis performed during the $375.5 \mathrm{~d}$ period corresponding to the 40 -string configuration of IceCube. We discuss here the impact of these limits on different models of neutrino emission from the Crab. Figure 5 summarizes 


\begin{tabular}{|c|c|c|c|c|c|c|c|c|c|c|c|}
\hline \multicolumn{2}{|c|}{ Spectrum } & \multicolumn{5}{|c|}{ Straight Cuts sample } & \multicolumn{5}{|c|}{ BDT sample } \\
\hline$E^{-\gamma}$ & $\begin{array}{l}\mathrm{E}_{\text {cutoff }} \\
(\mathrm{TeV})\end{array}$ & $n_{s}^{90 \%}$ & $\Phi_{\nu_{\mu}}^{90 \%}$ & $\begin{array}{c}\mathrm{E}_{\min } \\
(\mathrm{GeV})\end{array}$ & $\begin{array}{c}\mathrm{E}_{\max } \\
(\mathrm{GeV})\end{array}$ & $\mathcal{L}_{\nu_{\mu}}^{90 \%}$ & $n_{s}^{90 \%}$ & $\Phi_{\nu_{\mu}}^{90 \%}$ & $\begin{array}{c}\mathrm{E}_{\min } \\
(\mathrm{GeV})\end{array}$ & $\begin{array}{l}\mathrm{E}_{\max } \\
(\mathrm{GeV})\end{array}$ & $\mathcal{L}_{\nu_{\mu}}^{90 \%}$ \\
\hline$E^{-2}$ & - & 2.15 & 4.84 & $10^{3.3}$ & $10^{5.7}$ & 1.78 & 2.35 & 4.80 & $10^{3.1}$ & $10^{5.9}$ & 2.02 \\
\hline$E^{-2.7}$ & - & 2.41 & 32.6 & $10^{2.6}$ & $10^{4.9}$ & 6.0 & 2.90 & 26.3 & $10^{2.3}$ & $10^{4.7}$ & 6.77 \\
\hline$E^{-2}$ & 1 & 2.80 & 309 & $10^{2.4}$ & $10^{3.5}$ & 21.2 & 3.50 & 191 & $10^{2.3}$ & $10^{3.5}$ & 15.5 \\
\hline$E^{-2}$ & 100 & 2.25 & 8.59 & $10^{3.2}$ & $10^{5.0}$ & 1.98 & 2.51 & 8.06 & $10^{2.9}$ & $10^{4.9}$ & 2.05 \\
\hline$E^{-2}$ & 1000 & 2.20 & 5.52 & $10^{3.3}$ & $10^{5.6}$ & 1.79 & 2.34 & 5.31 & $10^{3.0}$ & $10^{5.5}$ & 1.86 \\
\hline
\end{tabular}

Table 2: Upper limits of the Crab Sep. 2010 flare using Neyman for both samples and different neutrino spectra including those with an exponential energy cut-off expressed as $E^{-\gamma} \exp \left(-E / E_{\text {cutoff }}\right)$ where $\mathrm{E}_{\text {cutoff }}$ is the energy cut-off. The number $n_{s}^{90 \%}$ is the limit in terms of number of signal events for a $90 \%$ confidence level and $\Phi_{\nu_{\mu}}^{90 \%}$ is the flux upper limit in units of $10^{-11} \mathrm{~cm}^{-2} \mathrm{~s}^{-1} \mathrm{TeV}^{-1}$ for a 9.28 days flaring interval. The resulting neutrino luminosity limit, $\mathcal{L}_{\nu_{\mu}}^{90 \%}$, is given in units of $10^{35} \mathrm{erg} \mathrm{s}^{-1}$ and it was calculated by integrating $d N^{90 \%} / d E \times E$ over the energy range from $E_{\min }$ to $E_{\max }$ to contain $90 \%$ signal of the spectrum and multiply by $4 \pi d^{2}$ where $d$ is the distance to the Crab Nebula $(d=1850 \mathrm{pc})$.

a number of different predicted fluxes described in the introduction of this paper and where the 40-string configuration limits stand [35]. Upper limits are defined as the $90 \%$ confidence level (CL) using the method from Feldman $\&$ Cousins [52]. The green line (solid) corresponds to the flux predicted in [12] based on the $\gamma$-ray spectrum measured by H.E.S.S. and the corresponding upper limit (dashed). The black line represents the estimated flux based on the resonant cyclotron absorption model proposed in [3] for the case of a wind Lorentz factor of $\Gamma=10^{7}$ and the most optimistic case of the effective target density. The red and blue lines represent the two predicted fluxes according to [7] for the cases of linear and quadratic proton acceleration respectively. The most optimistic version of this model (for both linear and quadratic proton acceleration) can be rejected with more than $90 \%$ CL using the time integrated data from 40 string configuration constraining this way the value of the charge depletion fraction.

\section{Conclusions}

Searches for neutrinos in coincidence with the Sep. 2010 Crab flare have been presented in this paper. The data used was taken with the 79-string 


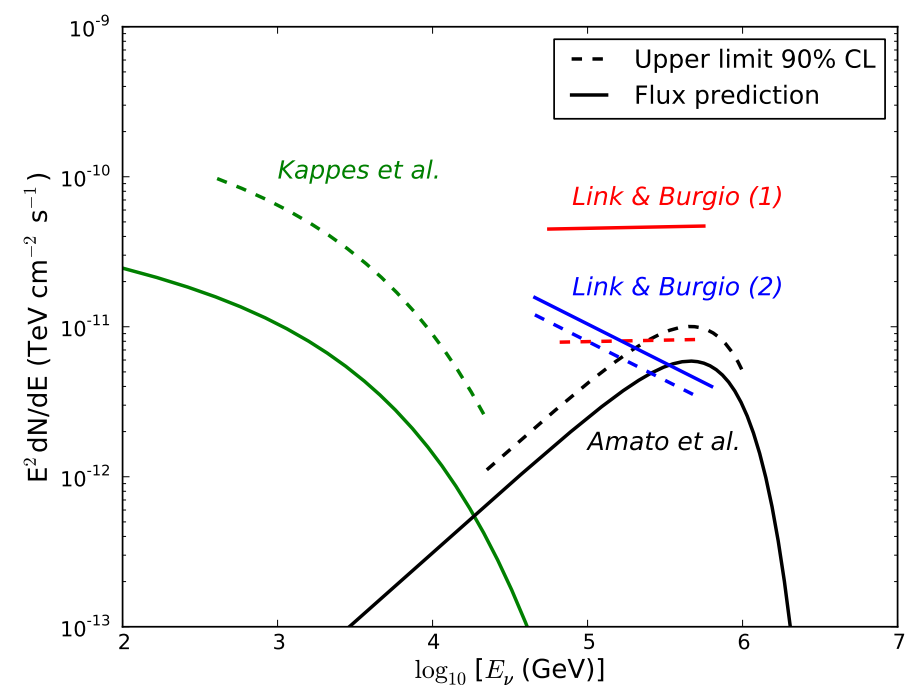

Figure 5: Predicted fluxes and upper limits based on the IceCube 40 string configuration on several models from the Crab. Solid lines indicate the predicted flux and dotted lines the corresponding upper limit for a 90\% CL. The green lines are the predicted flux and corresponding upper limit based on the model proposed in 12. The red and blue lines correspond to the model in [7] for the cases of linear (1) and quadratic (2) proton acceleration. The black line represents the estimated flux for the most optimistic model proposed in [3] based on resonant cyclotron absorption model and its corresponding upper limit. 
configuration of IceCube. This is the first analysis of data taken by this configuration and represents the first rapid response analysis of IceCube to an astronomical event such as the flaring of an otherwise steady standard candle source. Two different approaches of event selection have been followed. One using direct cuts on quality reconstruction variables and optimized for discovery for $E^{-2}$ neutrino spectra, and the other based on multivariate analysis and optimized for discovery at lower energies, important for galactic sources that have soft spectra with cut-offs at TeV energies. The two data sets however showed a background under-fluctuation during the time interval considered. The corresponding upper limits based on generic neutrino spectra have been shown for the flaring state of the Crab.

Assuming isotropic emission from the shock (even if this may not be the case for a highly relativistic pulsar wind) our limit for $E^{-2}$ corresponds to a neutrino luminosity constraint for the flare state of about $\sim 2 \times 10^{35} \mathrm{erg}$ $\mathrm{s}^{-1}$, and $\sim 1.5 \times 10^{36} \mathrm{erg} \mathrm{s}^{-1}$ if a neutrino cut-off of $1 \mathrm{TeV}$ is assumed. In both cases the resulting neutrino luminosity constraint is about $2-3$ orders of magnitude lower than the spin-down luminosity of the pulsar and comparable to the peak isotropic $\gamma$-ray luminosity $\sim 5 \times 10^{35} \mathrm{erg} \mathrm{s}^{-1}$ measured by AGILE [17] in the energy range from 0.1 to $10 \mathrm{GeV}$.

In addition to the flare analysis we calculated the current best limits set by IceCube on different models for neutrino emission from the Crab Nebula. These limits are based on the time-integrated analysis of IceCube with the 40string configuration of the detector. The upper regions of the most optimistic models can be rejected with more than 90\% CL providing useful constraints on adjustable parameters of these models. Taking the neutrino spectrum derived from the $\gamma$-ray observations from the Crab, the constraint in neutrino luminosity for the steady emission of the Crab is $\lesssim 1 \times 10^{35} \mathrm{erg} \mathrm{s}^{-1}$ which is a factor $\sim 1.7$ larger than the luminosity in $\gamma$-rays assuming the $\gamma$-ray spectrum measured in Ref. [13] integrated over the energy range between $400 \mathrm{GeV}-40 \mathrm{TeV}$.

In the future the IceCube detector will combine datasets from different detector configurations. When the different livetimes of the 40-string configuration data and the full detector will be summed, the sensitivity will improve by about a factor of five making this search more predictive. 


\section{Acknowledgements}

We acknowledge the support from the following agencies: U.S. National Science Foundation-Office of Polar Programs, U.S. National Science FoundationPhysics Division, University of Wisconsin Alumni Research Foundation, the Grid Laboratory Of Wisconsin (GLOW) grid infrastructure at the University of Wisconsin - Madison, the Open Science Grid (OSG) grid infrastructure; U.S. Department of Energy, and National Energy Research Scientific Computing Center, the Louisiana Optical Network Initiative (LONI) grid computing resources; National Science and Engineering Research Council of Canada; Swedish Research Council, Swedish Polar Research Secretariat, Swedish National Infrastructure for Computing (SNIC), and Knut and Alice Wallenberg Foundation, Sweden; German Ministry for Education and Research (BMBF), Deutsche Forschungsgemeinschaft (DFG), Research Department of Plasmas with Complex Interactions (Bochum), Germany; Fund

for Scientific Research (FNRS-FWO), FWO Odysseus programme, Flanders Institute to encourage scientific and technological research in industry (IWT), Belgian Federal Science Policy Office (Belspo); University of Oxford, United Kingdom; Marsden Fund, New Zealand; Japan Society for Promotion of Science (JSPS); the Swiss National Science Foundation (SNSF), Switzerland; A. Gro acknowledges support by the EU Marie Curie OIF Program; J. P. Rodrigues acknowledges support by the Capes Foundation, Ministry of Education of Brazil.

\section{References}

[1] J. J. Hester, Annual Review of Astronomy and Astrophysics 46 (2008) 127.

[2] C. A. Wilson-Hodge et al., When A Standard Candle Flickers, Astrop. J. 727 (2011) L40.

[3] E. Amato, D. Guetta and P. Blasi, Astron. Astrophys. 402 (2003) 827.

[4] W. Bednarek, Astron. Astrophys. 407 (2003) 1.

[5] Y.A. Gallant and J. Arons, Astrop. J. 435 (1994) 230.

[6] W. Bednarek and R.J. Protheroe, Phys. Rev. Lett. 79 (1997) 2616. 
[7] B. Link \& F. Burgio, Phys. Rev. Lett. 94 (2005) 181101.

[8] B. Link \& F. Burgio, Mon. Not. Roy. Astron. Soc. 371 (2006) 375.

[9] J. Alvarez-Muniz \& F. Halzen, Astrop. J. 576 (2002) L33.

[10] W. Bednarek et al., New Astron. Rev. 49 (2005) 1.

[11] W. Bednarek \& W. Idec, On the variability of the GeV and multi-TeV gamma-ray emission from the Crab Nebula, arXiv:1011.4176.

[12] A. Kappes et al., Astrophys. J. 656 (2007) 870.

[13] F. Aharonian et al., Astron. Astrophys. 457 (2006) 899.

[14] F. Aharonian et al., Astrop. J. 539 (2000) 317.

[15] J. Albert et al., Astrop. J. 674 (2008) 1037.

[16] M. Tavani et al., http://www.astronomerstelegram.org/?read=2855

[17] M. Tavani et al., Science, 331 (2011) 736.

[18] A. A. Abdo et al, http://www.astronomerstelegram.org/?read=3284

[19] A. Tennant et al., http://www.astronomerstelegram.org/?read=2882

[20] C. Ferrigno et al., http://www.astronomerstelegram.org/?read=2856

[21] C. B. Markward, http://www.astronomerstelegram.org/?read=2858

[22] N. Shaposhnikov, http://www.astronomerstelegram.org/?read=2872

[23] G. Aielli et al., http://www.astronomerstelegram.org/?read=2921

[24] A. A. Abdo et al, Science, 331, (2011) 739.

[25] M. Mariotti et al., http://www.astronomerstelegram.org/?read=2967

[26] R. A. Ong et al., http://www.astronomerstelegram.org/?read=2968

[27] J. Braun et al., Astropart. Phys. 33 (2010) 175. 
[28] R. Abbasi et al., Time-Dependent Searches for Point Sources of Neutrinos with the 40-String and 22-String Configurations of IceCube, submitted to ApJ, arXiv:1104.0075.

[29] R. Abbasi et al. [IceCube Coll.], Nucl. Inst. Meth. A 618 (2010) 139.

[30] R. Abbasi et al. [IceCube Coll.], The Design and Performance of IceCube DeepCore, in preparation.

[31] R. Abbasi et al. [IceCube Coll.], Nucl. Inst. Meth. A 601 (2009) 294.

[32] S. Tilav et al. [IceCube Coll.], Atmospheric Variations as observed by IceCube, arXiv:1001.0776.

[33] M. Ackermann, M. and E. Bernardini for the IceCube, An investigation of seasonal variations in the atmospheric neutrino rate with the AMANDA-II neutrino telescope, astro-ph/0509330.

[34] J. Ahrens et al. [AMANDA Coll.], Nucl. Instr. Meth. A 524 (2004) 169.

[35] R. Abbasi et al. [IceCube Coll.], Time-Integrated Searches for Point-like Sources of Neutrinos with the 40-String IceCube Detector, Astrop. J. 732 (2011) 18.

[36] A. Franckowiak et al. [IceCube Coll.] Optical follow-up of high-energy neutrinos detected by IceCube, arXiv:0909.0631; M. Ackermann et al. [IceCube Coll.] Neutrino Triggered Target of Opportunity (NToO) test run with AMANDA-II and MAGIC, arXiv:0709.2640.

[37] H. Cramér, Mathematical Methods of Statistics, Princeton Univ. Press (1946 and C. R. Rao, Information and the accuracy attainable in the estimation of statistical parameters, Bulletin of the Calcutta Mathematical Society 37 (1945) 81.

[38] R. Abbasi et al. [IceCube Coll.], Phys. Rev. Lett. 102 (2009) 201302.

[39] S. S. Kerthi et al., Neural Comp. 13 (2001) 637.

[40] A. Gazizov and M.P. Kowalski, Comput. Phys. Commun. 172 (2005) 203.

[41] H. L. Lai et al., Eur. Phys. J. C12 (2000) 375. 
[42] G. D. Barr et al., Phys. Rev. D70 (2004) 023006.

[43] M. Honda et al., Phys. Rev. D75 (2007) 043006.

[44] A. D. Martin, M. G. Ryskin and A. M. Stasto, Acta Phys. Polon. B34 (2003) 3273.

[45] R. Enberg, M. H. Reno and I. Sarcevic, Phys. Rev. D78 (2008) 043005.

[46] D. Heck et al., CORSIKA: A Monte Carlo code to simulate extensive air showers FZKA-6019 (1998).

[47] E.-J. Ahn et al., Phys. Rev. D80 (2009) 094003.

[48] D. Chirkin and W. Rhode, Muon Monte Carlo: A high-precision tool for muon propagation through matter, hep-ph/0407075.

[49] R. Abbasi et al. [IceCube Coll.], Study of South Pole ice transparency with IceCube flashers, in preparation.

[50] M. Ackermann et al., J. Geophys. Res. 111 (2006) D13203.

[51] J. Lundberg et al., Nucl. Instrum. Meth. A581 (2007) 619.

[52] G. J. Feldman and R. D. Cousins. Phys. Rev. D57 (1998) 3873. 